\title{
IMPORTANCIA AGROBIOTECNOLÓGICA DE LA ENZIMA ACC DESAMINASA EN RIZOBACTERIAS, UNA REVISIÓN
}

\author{
AGROBIOTECHNOLOGICAL IMPORTANCE OF THE ACC DEAMINASE IN \\ RHIZOBACTERIA, A REVIEW
}

\author{
Rosalba Esquivel-Cote ${ }^{\star \star}$, Marina Gavilanes-Ruiz ${ }^{2}$, Rocío Cruz-Ortega ${ }^{3}$ y Pilar Huante ${ }^{4}$
}

\begin{abstract}
${ }^{1}$ Laboratorio de Microbiología Experimental, Departamento de Biología, ${ }^{2}$ Departamento de Bioquímica de la Facultad de Química, Conjunto E, ${ }^{3}$ Laboratorio de Alelopatía, ${ }^{4}$ Laboratorio de Ecofisiología Vegetal, Departamento de Ecología Funcional, Instituto de Ecología, Universidad Nacional Autónoma de México (UNAM). Av. Universidad 3000, Delegación Coyoacán. 04510, México, DF.
\end{abstract}

${ }^{*}$ Autor para correspondencia (rosesquivel_cote@hotmail.com)

\section{RESUMEN}

La rizosfera es la región del suelo con la mayor actividad microbiana, con una gran riqueza en nutrimentos, y en donde planta y microorganismo interaccionan mutuamente para su beneficio. Las rizobacterias promotoras del crecimiento vegetal (PGPR, por sus siglas en inglés), utilizan uno o varios mecanismos de acción para favorecer este crecimiento, ya sea estimulando la absorción de nutrimentos o evitando la acción de microorganismos patógenos. La actividad de la enzima desaminasa del ácido 1-aminociclopropano-1-carboxílico (ACC) o ACC desaminasa es un mecanismo que utilizan algunas PGPR para promover el crecimiento de plantas influenciadas por el estrés ambiental, la cual les trae dos ventajas importantes: disminuir las concentraciones de etileno en la planta e incrementar la disponibilidad de amonio en la rizosfera. Con ello, la actividad de la enzima ACC desaminasa mejora la nutrición vegetal y la resistencia a factores de estrés. Es posible que el uso de PGPR que contienen ACC desaminasa permita mejorar sistemas agrícolas de ambientes áridos o salinos o con problemas de contaminación por metales pesados. En esta revisión se reseñan aspectos básicos de la interacción entre las PGPR y la planta, y se analiza el mecanismo de acción de la enzima ACC desaminasa y su aplicación en problemas agrícolas y de biorremediación.

Palabras clave: ACC desaminasa, etileno, inoculantes, PGPR, rizobacterias.

\section{SUMMARY}

The rhizosphere is a nutrient-rich soil region with important levels of microbial activity. In this zone, plants and some microorganisms such as plant growth-promoting rhizobacteria (PGPR) mutually interact for their benefit. These bacteria use one or several mechanisms to favor plants: either stimulating nutrients absorption or avoiding the action of pathogenic microorganisms. The activity of the 1-aminocyclopropane-1-carboxylic acid (ACC) deaminase is a strategy that some PGPR use to promote plant growth under specific environmental stress. This enzymatic activity provides two important advantages for plants: reducing ethylene concentration in the plant, and increasing ammonium availability at the rhizosphere. In this way, the activity of the ACC deaminase improves plant nutrition and resistance to stress factors. ACC deaminase-containing PGRP can be used to improve agricultural systems under arid and salt conditions, and at polluted environments with heavy metals. This review examined the basic aspects of the interaction between the PGPR and the plant and the ACC deaminase action mechanism. A compilation of the reported
PGPR species and their application in agricultural and bioremediation is also presented.

Index words: ACC deaminase, ethylene, inoculants, PGPR, rhizobacteria.

\section{INTRODUCCIÓN}

La rizosfera es un sistema dinámico donde se detecta la máxima actividad microbiana, en el cual las interacciones y la comunicación entre raíz y microorganismo juegan un papel muy importante en el mantenimiento del crecimiento y productividad vegetal (Curl y Truelove, 1986). La interacción entre los microorganismos y las plantas puede ser benéfica (promueve el crecimiento vegetal), perjudicial (provoca enfermedades) o neutra (Zahir et al., 2004). Dentro del tipo benéfico, el mutualismo es la interacción más importante de la rizosfera, y se lleva a cabo entre las plantas y las bacterias asociadas a las raíces (rizobacterias) (Badri et al., 2009).

Las rizobacterias que tienen un efecto benéfico en la planta son usualmente referidas como promotoras del crecimiento vegetal o PGPR, por sus siglas en inglés (plant growth promoting rhizobacteria) (Davison, 1988). Muchas de éstas han sido propagadas y usadas como inoculantes bacterianos, principalmente para mejorar la producción y el rendimiento de cultivos agrícolas. Las PGPR promueven el crecimiento vegetal mediante dos tipos de mecanismos: indirectos y directos, o una combinación de ambos.

Los mecanismos indirectos se caracterizan porque las PGPR ocasionan la disminución o eliminación de microorganismos fitopatógenos (hongos, bacterias y nemátodos), ya sea a través de la producción de sustancias antimicrobianas, de sideróforos, de enzimas líticas, o una combinación de éstas; por competencia de nutrimentos o de espacio en el nicho ecológico, así como por estimulación de las defensas naturales de la planta mediante mecanismos 
conocidos como resistencia sistémica inducida (RSI). Esta última induce la resistencia de tejidos sistémicos al ataque por fitopatógenos mediante la emisión de compuestos orgánicos volátiles, de ácido jasmónico, de ácido salicílico y del etileno que participan en la protección de las plantas a diferentes enfermedades (Kloepper et al., 1993; Glick, 1995; Shah, 2009).

Los mecanismos directos incrementan la disponibilidad de nutrimentos en la rizosfera al influir en el metabolismo de las plantas y mejorar su nutrición. Estos mecanismos son: fijación de nitrógeno; síntesis de fitohormonas (auxinas, giberelinas, citocininas), vitaminas y enzimas; solubilización de fósforo inorgánico y mineralización de fosfato orgánico; oxidación de sulfuros; incremento en la permeabilidad de la raíz; producción de nitritos; acumulación de nitratos; y reducción de la toxicidad por metales pesados y de la actividad de la enzima ACC desaminasa (Glick 1995; Dobbelaere et al., 2003). A este respecto, se ha propuesto que en el efecto que Azospirillum ejerce sobre la planta intervienen múltiples mecanismos que actúan simultánea y sucesivamente para promover el crecimiento vegetal (Bashan y Levanony, 1990; Bashan y Dubrovsky, 1996).

Las plantas viven en un ambiente cambiante y frecuentemente impredecible. Como organismo sésil, las plantas deben hacer frente a todo tipo de perturbaciones que afecten su hábitat en particular. Los factores ambientales son clasificados como bióticos y abióticos. Los factores bióticos son los que involucran la presencia de agentes biológicos que dañan a las plantas, como los herbívoros (insectos, mamíferos) y los microorganismos patógenos (bacterias, hongos, virus). Como factores abióticos se consideran: concentración de $\mathrm{CO}_{2}$, temperatura, disponibilidad de agua, y características del suelo como salinidad, contenido de macro y micronutrimentos, y presencia de contaminantes (metales pesados e hidrocarburos).

En respuesta a tales factores adversos las plantas incrementan su biosíntesis de etileno, lo que induce ciertos cambios característicos en la planta, como detener la elongación de la raíz e inducir la producción y elongación de raíces adventicias, acelerar la senescencia de flores, promover la abscisión de flores y frutos y, finalmente, provocar la muerte de la planta (Abeles et al., 1992; Des Marais y Juenger, 2010). No obstante, las plantas han desarrollado diversas estrategias que les han permitido adaptarse o sobrellevar el estrés ambiental, donde la intervención de los microorganismos tiene un papel muy importante.

En 1998 se sugirió que algunas rizobacterias promueven el crecimiento vegetal mediante la disminución de los niveles de etileno en plantas, a través de la acción de una enzima que desamina al precursor inmediato del etileno, el ácido 1-aminociclopropano-1-carboxílico (ACC): tal enzima es la ACC desaminasa (Glick et al., 1998). El objetivo del presente trabajo es revisar la importancia agrobiotecnológica del uso de rizobacterias para promover el crecimiento vegetal mediante la enzima ACC desaminasa, como inoculantes de plantas de interés agrícola y en la fitorremediación.

\section{BIOSÍNTESIS DEL ETILENO EN PLANTAS}

El sitio de síntesis y acción del etileno está localizado en cualquier tejido vegetal, de donde es liberado fácilmente y difundido a través de los espacios intercelulares y por fuera de ellos, dada su naturaleza gaseosa. Su producción está regulada por un amplio número de factores, como temperatura, luz, gravedad, nutrición y otras fitohormonas (Abeles et al., 1992). En plantas superiores, el etileno se sintetiza a partir del aminoácido $L$-metionina que se encuentra en los exudados de la raíz.

Como se ilustra en la Figura 1, la metionina es activada por el ATP para formar $S$-adenosilmetionina (SAM) (2) a través de la reacción catalizada por la enzima SAM sintetasa (EC 2.5.1.6) (1), la cual es inducida durante la senescencia o por condiciones de estrés ambiental. SAM es convertida a ácido 1-aminociclopropano-1-carboxílico (ACC) (4) mediante la enzima ACC sintasa (EC 4.4.1.14) (3) que utiliza piridoxal-5-fosfato como cofactor. Dicha enzima es estimulada por fitohormonas como las auxinas (AIA) y citocininas (Z) y por el propio etileno. Finalmente, a partir del ACC el etileno es sintetizado por la enzima dependiente de hierro, ACC oxidasa (EC 1.14.17.4) (5), paso en el que además del etileno también se producen $\mathrm{CO}_{2}$ y HCN (Ververidis y John, 1991). Esta reacción es dependiente del oxígeno y su síntesis se incrementa con el aumento de $\mathrm{CO}_{2}$. El hierro $\left(\mathrm{Fe}^{++}\right)$actúa como cofactor y el ascorbato como cosustrato. Las enzimas ACC sintasa y ACC oxidasa tienen un corto tiempo de vida media y existen en bajas concentraciones en muchos tejidos vegetales (Hontzeas et al., 2004). Las plantas pueden convertir también el ACC en $\mathrm{N}$-malonil-ACC mediante la ACC $N$-malonil transferasa.

\section{ACCIÓN DE LA ENZIMA ACC DESAMINASA EN LA BIOSÍNTESIS DEL ETILENO}

La función de la enzima ACC desaminasa es convertir el ACC en $\alpha$-cetobutirato ( $\alpha$-CB) y amonio (6) (Figura 1) (Kende, 1993; Bleecker y Kende, 2000). Tanto el amonio como el $\alpha$-CB son metabolitos comunes en plantas y otros organismos.

Glick et al. (1998) plantearon un modelo mediante el cual las PGPR disminuyen los niveles de etileno en plantas y a su vez estimulan el crecimiento vegetal. Según el modelo ilustrado en la Figura 2, las bacterias que se encuentran adheridas 


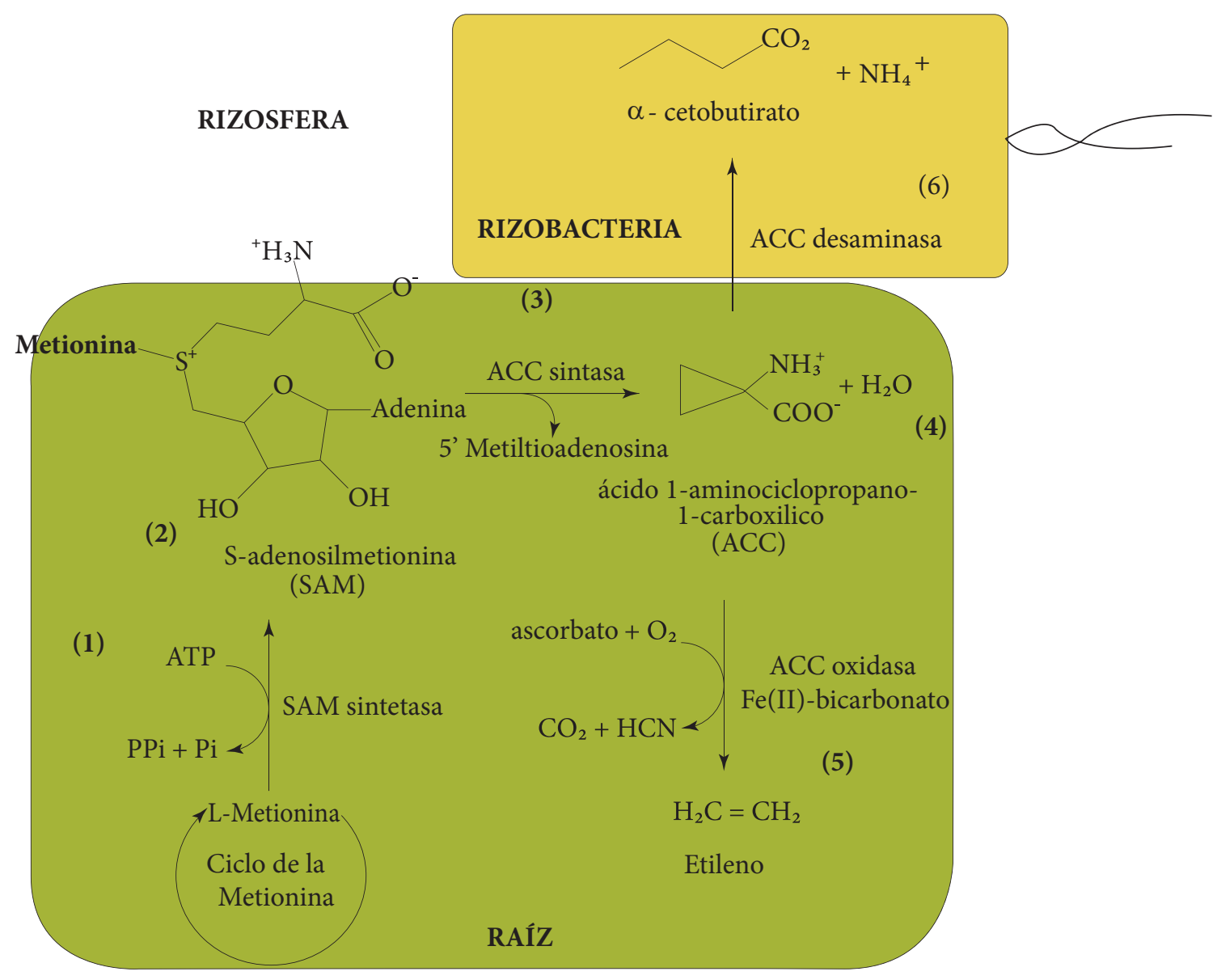

Figura 1. Reacción de la enzima desaminasa del ácido 1-aminociclopropano-1-carboxílico (ACC) en el contexto de las reacciones de síntesis y degradación del ACC, precursor del etileno (modificado de Glick et al., 1998).

a la superficie de las semillas o de las raíces emplean al triptófano contenido en los exudados para sintetizar y liberar ácido indol-3-acético (AIA). Este AIA exógeno junto con el AIA endógeno de las plantas estimula la proliferación y elongación celular vegetal, y ambos inducen la síntesis de la enzima ACC sintasa que convierte a SAM en ACC (Kende, 1993). Finalmente, el ACC presente en los exudados es hidrolizado en $\alpha$-cetobutirato y amonio, y este último es utilizado por plantas y bacterias rizosféricas como una alternativa más de fuente de nitrógeno.

Para mantener el equilibrio entre los niveles de ACC interno y externo, las plantas exudan más ACC y como resultado disminuye la biosíntesis de etileno dentro de la planta (Penrose y Glick, 2001). En apoyo a este modelo, Madhaiyan et al. (2006) reportaron que la inoculación de plantas de canola (Brassica napus, Brassicaceae) con cepas del género bacteriano Methylobacterium incrementa la concentración tanto de AIA y citocininas (trans-zeatin-ribosa e isopenteniladenosina), por lo que también se incrementa la actividad de la enzima ACC sintasa.
Tales hallazgos indican que la promoción del crecimiento vegetal es estimulada no sólo por un decremento en el contenido de etileno, sino también por generación de amonio producido a partir del ACC por rizobacterias que poseen a la ACC desaminasa (Glick et al., 1998; Glick et al., 2007). Con esta estrategia las plantas disponen de un recurso extra de nutrimentos, y los microorganismos de la rizosfera tienen una alternativa más en la generación del nitrógeno para su supervivencia. Con ello las PGPR capaces de producir la enzima ACC desaminasa (PGPR-ACC desaminasa) incrementan su proliferación en los sitios de colonización de las raíces y son favorecidas ante la competencia con otros microorganismos. Lo último ha permitido que las PGPR-ACC desaminasa se empleen frecuentemente como inoculantes de plantas cultivadas en condiciones desfavorables, para mejorar su crecimiento (Belimov et al., 2001). Con todos estos elementos a su favor, la enzima ACC desaminasa ha sido propuesta como un elemento clave en la asociación planta-microorganismo (Hontzeas et al., 2004). 


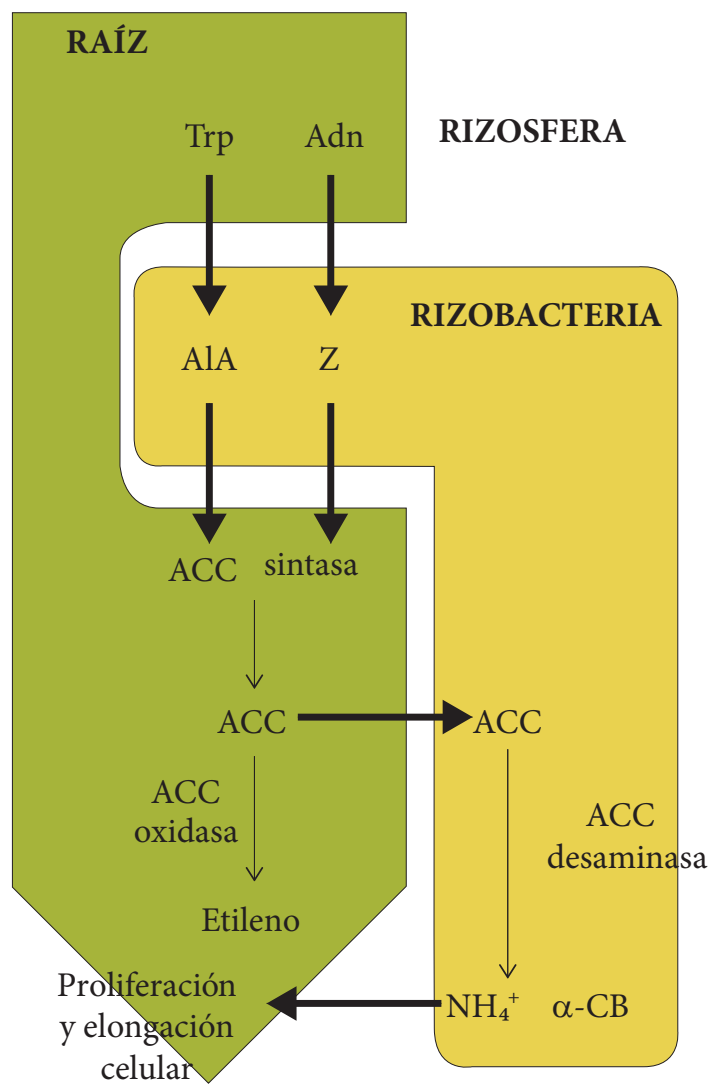

Figura 2. Modelo del mecanismo de la enzima desaminasa del ácido 1-aminociclopropano1-carboxílico (ACC) mediante el cual las rizobacterias promotoras del crecimiento vegetal disminuyen los niveles de etileno en plantas (modificado de Glick et al., 1998).

\section{ACTIVIDAD ENZIMÁTICA DE LA ACC DESAMINASA}

La actividad enzimática de la ACC desaminasa es inducida por niveles bajos (alrededor de $100 \mathrm{nM}$ ) del sustrato ACC, aunque no presenta una afinidad alta por el mismo. Lo anterior presenta dos grandes consecuencias: la primera es que la enzima ACC oxidasa (la cual cataliza la formación de etileno a partir del ACC) tiene mayor afinidad por el ACC que la enzima ACC desaminasa; por tanto, una forma para que ésta pueda competir efectivamente con la ACC oxidasa por el ACC, es mediante un aumento en la cantidad de ACC desaminasa disponible. Para competir ventajosamente por el sustrato con la ACC oxidasa, la ACC desaminasa debe tener valores de 100 a 1000 veces mayores que los de la oxidasa.

Dada la baja afinidad de la desaminasa por el ACC, las concentraciones milimolares fisiológicas de su sustrato permiten que la velocidad de la enzima sea proporcional a la magnitud de los cambios de concentración del ACC. Adicionalmente, la inducción de la síntesis de la enzima por concentraciones bajas de ACC garantiza la utilización eficiente de este sustrato (Glick, 2005). Es importante considerar que los niveles de ACC desaminasa en diferentes microorganismos varían ampliamente, lo cual implica diversas formas de regulación de la actividad de la enzima, ya sea a nivel de la enzima misma o de la expresión de su gen.

\section{GENÉTICA DE LA ENZIMA ACC DESAMINASA}

La enzima ACC desaminasa es codificada por el gen acdS, el cual ha sido identificado con una frecuencia relativamente alta en varios géneros bacterianos presentes en la rizosfera de diversos tipos de suelos en varias zonas geográficas (Glick et al., 1995; Belimov et al., 2001). Su expresión es co-regulada por las proteínas acdR y acdB (Blaha et al., 2006; Cheng et al., 2008; Prigent-Combaret et al., 2008). La secuencia de nucleótidos que forman el gen acdS no es muy similar entre géneros bacterianos, ya que varía incluso entre cepas del mismo género; así, dentro de un mismo género o especie existen cepas que presentan actividad de la enzima ACC desaminasa y otras no. Por ejemplo, mientras que cepas de Azospirillum lipoferum presentan el gen acdS 
(Esquivel-Cote et al., 2010), otras cepas de A. brasilense no lo tienen (Holguín y Glick, 2001; Blaha et al., 2006).

Estas observaciones, y los resultados obtenidos mediante un análisis filogenético de genes acdS, realizados por Hontzeas et al. (2005), sugieren que estos genes podrían ser adquiridos mediante transferencia horizontal $y$, además, que los genes que codifican para la ACC desaminasa no permanecen siempre en la parte integral del cromosoma del ADN del microorganismo, sino que se presentan relativamente estables a nivel de plásmido (Glick et al., 2007).

Algunos genes acdS identificados en bacterias se han aislado y usado para la transformación genética de otras bacterias y de plantas (Holguin y Glick, 2003). En bacterias la transformación se ha realizado para aumentar el número de mecanismos promotores del crecimiento vegetal, lo que se ha logrado en cepas de Sinorhizobium meliloti, Mesorhizobium lotti y Azospirillum brasilense (Holguín y Glick, 2001; Ma et al., 2004; Nukui et al., 2006). En plantas el gen acdS ha sido utilizado para la transformación de tomate (Solanum lycopersicum, Solanaceae), a fin de disminuir el proceso de maduración del fruto (Klee, 1993); y de canola (Brassica napus, Brassicaseae), para incrementar su tolerancia a metales pesados como cadmio, cobalto, cobre, níquel, plomo y zinc (Grichko et al., 2000; Stearns et al., 2005).

A pesar de que el compuesto ACC se ha identificado dentro de los exudados de la raíz, la enzima ACC desaminasa no había sido identificada en plantas. Fue hasta el año 2009 que se reportó su presencia en extractos de plantas de Arabidopsis (Brassicaseae) y de tomate (McDonnell et al., 2009; Plett et al., 2009). Lo anterior sugiere que existe una transferencia de genes acdS entre plantas y bacteria, sean estas últimas de efecto promotor del crecimiento o de virulencia para la planta (Blaha et al., 2006).

\section{EFECTO DE LA INOCULACIÓN DE PLANTAS CON PGPR QUE SINTETIZAN LA ENZIMA ACC DESAMINASA}

El efecto de la inoculación en el crecimiento de las plantas con bacterias que sintetizan la enzima ACC desaminasa depende significativamente de varios factores, como del estatus nutrimental de la planta o del abuso de las prácticas agrícolas (Morgan y Drew, 1997; Belimov et al., 2002; Castro-Sowinski et al., 2007).

Es importante mencionar que el nivel de la actividad de la ACC desaminasa no tiene un efecto proporcional en el crecimiento vegetal. Es decir, las bacterias cultivadas en presencia de ACC y con niveles bajos de actividad ACC desaminasa $\left(\geq 20 \mathrm{nmol} \alpha-\mathrm{CB} \mathrm{mg}^{-1} \mathrm{~h}^{-1}\right)$ pueden promover significativamente el crecimiento vegetal, en tanto que bac- terias con niveles altos de actividad ACC desaminasa (400 nmol $\left.\alpha-C B ~ \mathrm{mg}^{-1} \mathrm{~h}^{-1}\right)$ no necesariamente tienen un efecto promotor (Penrose y Glick, 2003). A continuación se mencionan algunos ejemplos de los efectos benéficos de la inoculación de plantas en condiciones de estrés con PGPR con actividad ACC desaminasa (PGPR-ACC desaminasa), nativas o transformadas.

\section{Estrés hídrico por salinidad}

La bacteria Pseudomonas fluorescens TDK1 proporciona resistencia al estrés salino en plantas de cacahuate (Arachis hypogea, Fabaceae), y con ello incrementa la producción del cultivo (Saravanakumar y Samiyappa, 2007). En pepino (Cucumis sativus L.) la interacción entre la cepa UW4 de Pseudomonas putida y el hongo micorrízico Gigaspora rosea BEG9 tiene un efecto sinérgico en el crecimiento de plantas crecidas en condiciones de estrés por salinidad (Gamalero et al., 2010). La bacteria Achromobacter piechaudii ARV8 favorece la eficiencia del uso del agua, ya que reduce la producción de etileno e incrementa el peso fresco y seco de plantas de tomate y pimiento (Capsicum annuum, Solanaceae) en presencia de altas concentraciones de $\mathrm{NaCl}$. Así mismo, las bacterias halotolerantes como Arthrobacter nicotianae (RSA68), Bacillus stratosphericus (RS233), Corynebacterium variabile (RS665), Exiguobacterium acetylicum (RS343), Halomonas neptunia (ES11E), Oceanimonas smirnovii (RS231), Planococcus rifietensis (RS224) y Zhihengliuella alba (RS111) incrementan el crecimiento y la resistencia a la salinidad de plantas de pimiento (Siddikee et al., 2010). En un ambiente con escasez de agua, A. piechaudii mejora la recuperación de las plantas de tomate cuando el riego se restablece (Mayak et al., 2004a; Mayak et al., 2004b).

\section{Estrés anaeróbico}

Las cepas Enterobacter cloacae UW4, E. cloacae CAL2, y Pseudomonas putida ATCC17399/pRKACC (genéticamente transformada), protegen eficazmente a plantas de tomate contra el estrés por inundación; promueven el desarrollo vegetal, aumentan la cantidad de clorofila en las hojas y reducen sustancialmente la producción foliar de etileno (Grichko y Glick, 2001a; 2001b).

\section{Ataque de plantas por fitopatógenos}

La reducción de la biosíntesis del etileno en plantas de tomate transformadas con el gen de la enzima ACC desaminasa de la bacteria Enterobacter cloacae UW4, disminuye los síntomas de la enfermedad causada por el hongo fitopatógeno Verticillium (Robison et al., 2001). La bacteria Pseudomonas brassicacearum Am3 puede mostrar tanto propiedades patogénicas como de PGPR en su interacción con plantas de tomate (Belimov et al., 2007). 


\section{Contaminación del suelo por metales pesados e hidrocarburos}

Las plantas de canola (Brassica napus) y tomate inoculadas con Kluyvera ascorbata SUD165 crecen en altas concentraciones de cloruro de níquel porque disminuyen los niveles de etileno sintetizado en respuesta al estrés inducido por la presencia del metal (Burd et al., 1998; 2000). Las plantas transgénicas de canola que expresan el gen que codifica para la enzima ACC desaminasa de la bacteria Enterobacter cloacae UW4, presentan la capacidad de crecer en altas concentraciones de arsenato y de níquel en el suelo, además de acumular este metal en el tejido vegetal.

La bacteria Enterobacter cloacae CAL2 facilita la promoción del desarrollo de plantas de canola transformadas y no transformadas, en presencia de arsenato (Nie et al., 2002). En tanto que cuando son inoculadas con Pseudomonas putida HS-2, aislada de suelos contaminados con níquel, las plantas incrementan significativamente su biomasa y favorecen la absorción de níquel en raíces y follaje. Estos resultados han conducido a catalogar a la cepa HS-2 como una candidata potencial para ser usada tanto en protocolos para biorremediación como para promover el crecimiento vegetal (Rodríguez et al., 2008).

Del mismo modo, bacterias endófitas de plantas tolerantes al cobre (Pantoea agglomerans Jp3-3 y Pseudomonas thivervalensis Y1-3-9) incrementan el crecimiento vegetal y la acumulación de cobre de Brassica napus (Zhang et al., 2011). La bacteria Pseudomonas asplenii AC, genéticamente transformada, incrementa la germinación y el desarrollo vegetal de plantas de carrizo (Phragmites australis, Poaceae) en presencia de cobre e hidrocarburos aromáticos como creosota, un hecho importante para la fitorremediación porque el carrizo es una planta tradicionalmente usada para depurar aguas residuales por ser tolerante a metales pesados (Reed et al., 2005). Plantas transgénicas de tomate que expresan la enzima ACC desaminasa (controladas por el promotor PRB-1b), pueden acumular una gran cantidad de metal dentro del tejido vegetal y disminuir el efecto deletéreo de metales pesados como $\mathrm{Cd}, \mathrm{Co}, \mathrm{Cu}, \mathrm{Mg}, \mathrm{Ni}, \mathrm{Pb}$ o $\mathrm{Zn}$, en el desarrollo de la planta, en comparación con las plantas no transgénicas (Grichko et al., 2000).

Especies como Alcaligenes xylosoxidans, Alcaligenes sp., Bacillus pumilus, Pseudomonas brassicacearum, Pseudomonas marginalis, Pseudomonas oryzihabitans, Pseudomonas putida, Pseudomonas sp., Rhodococcus sp. y Variovorax paradoxus, estimulan la germinación de plántulas de mostaza parda (Brassica juncea, Brassicaceae) y canola. Además, estas bacterias son tolerantes a la toxicidad de cadmio y estimulan la elongación de la raíz en plántulas de canola (Belimov et al., 2001). Bacterias modificadas genéticamen- te para expresar la actividad ACC desaminasa mejoran la degradación de tolueno en plantas (Glick, 2004). Es decir, el uso de bacterias que producen la enzima ACC desaminasa han tenido impacto positivo en la fitorremediación (Glick, 2003; Glick, 2004; Arshad et al., 2007; Jing et al., 2007).

\section{Deficiencia nutrimental y aplicación de fertilizantes}

Algunas PGPR que tienen la capacidad de sintetizar la enzima ACC desaminasa favorecen la supervivencia de plántulas, al protegerlas contra los efectos inhibitorios provocados por altas o bajas concentraciones de nutrimentos (Abeles et al., 1992; Lynch y Brown, 1997; Belimov et al., 2002; Shaharoona et al., 2008). La bacteria Pseudomonas fluorescens biotipo $\mathrm{G}\left(\mathrm{N}_{3}\right)$ mejora el desarrollo y rendimiento de plantas de maíz (Zea mays, Poaceae) en presencia de fertilizante nitrogenado aplicado en dosis óptimas (Saharoona et al., 2006). En presencia de dosis óptimas o apropiadas de fertilizante nitrogenado, la inoculación con PGPR capaces de sintetizar la enzima ACC desaminasa mejora el desarrollo y rendimiento de las plantas inoculadas (Tahir et al., 2006; Shaharoona et al., 2006; Shaharoona et al., 2008). Un importante efecto de la inoculación con PGPR-ACC desaminasa es la reducción en la aplicación de nitrógeno inorgánico en los sistemas agrícolas.

\section{CONCLUSIONES}

Las bacterias del suelo que sintetizan a la enzima ACC desaminasa juegan un papel importante en el desarrollo de plantas expuestas a factores de estrés biótico y abiótico, al facilitar la absorción de nutrimentos y agua, e incrementar así la biomasa y el rendimiento de los cultivos. Los mecanismos de resistencia al estrés son diversos y no están totalmente entendidos, de ahí que el estudio de la relación de éstos con la participación de las bacterias promotoras del crecimiento vegetal que sintetizan la enzima ACC desaminasa (PGPR-ACC desaminasa) sea aún incipiente.

Así mismo, es importante enfatizar que en la diversidad de opciones para promover el crecimiento vegetal, la contribución de un mecanismo individual podría tener menos relevancia que la acción de mecanismos múltiples.

\section{PERSPECTIVAS}

Debido a que el conocimiento de los mecanismos que emplean las PGPR para promover el crecimiento vegetal es aún limitado, tanto a nivel básico como en su aplicación, es necesario realzar la importancia de fortalecer, principalmente en México y demás países en vías de desarrollo, en especial el estudio de las bacterias PGPR-ACC desaminasa con amplio espectro de colonización de especies vegetales. Estos 
estudios contribuirían a que estos microorganismos puedan ser empleados como biofertilizantes o fitoestimulantes eficaces en el área agrícola, y así poder mejorar la nutrición y la resistencia de los cultivos a la sequía, la salinidad y a las altas o bajas temperaturas, así como una disminución o inclusive un reemplazo del uso de fertilizantes químicos contaminantes, lo que traería un beneficio económico a los productores.

También es de relevancia la introducción de nuevas especies de PGPR-ACC desaminasa en estrategias de fitorremediación de suelos contaminados con metales pesados, para incrementar la capacidad remediadora de plantas o bien para reducir la fitotoxicidad de los contaminantes del suelo, ya que en estos ámbitos se han logrado éxitos en la recuperación de suelos contaminados con metales pesados.

El presente escrito surge como un esfuerzo para difundir en nuestro idioma, un área de la investigación científica que aún falta desarrollar en México.

\section{AGRADECIMIENTOS}

A la M. en C. Rosa María Ramírez Gama y a la M. en C. Guadalupe Tsuzuki Reyes por la revisión y crítica al manuscrito. Al CONACYT por el apoyo otorgado mediante la beca 181624 para la realización de estudios de doctorado de la primera autora, y por el financiamiento a través del proyecto 101521. Y a la Dirección General de Asuntos del Personal Académico, UNAM, por el financiamiento mediante el proyecto PAPIIT IN210812.

\section{BIBLIOGRAFÍA}

Abeles F B, P W Morgan, M E Jr Saltveit (1992) Ethylene in Plant Biology. 2a. ed. Academic Press, San Diego, C.A. 414 p.

Arshad M, M Saleem, S Hussain (2007) Perspectives of bacterial ACC deaminase in phytoremediation. Trends Biotech. 25:356-362.

Badri D V, T L Weir, D Van der Lelie, J M Vivanco (2009) Rhizosphere chemical dialogues: plant-microbe interactions. Curr. Op. Biotech. 20:642-650.

Bashan Y, H Levanony (1990) Current status of Azospirillum inoculation technology: Azospirillum is a challenge for agriculture. Can. J. Microbiol. 36:591-608.

Bashan Y, J G Dubrovsky (1996) Azospirillum spp. participation in dry matter partitioning in grasses at the whole plant level. Biol. Fertility Soil 23:435-440.

Belimov A A, V I Safronova, T A Sergeyeva, T N Egorova, V A Matveyeva, V E Tsyganov, A Y Borisov, I A Tikhonovich, C Kluge, A Preisfeld, K J Dietz, V V Stepanok (2001) Characterization of plant growth promoting rhizobacteria isolated from polluted soils and containing 1-aminocyclopropane-1-carboxylate deaminase. Can. J. Microbiol. 47:642-652.

Belimov A A, V I Safronova, T Mimura (2002) Response of spring rape (Brassica napus var. oleifera L.) to inoculation with plant growth promoting rhizobacteria containing 1-aminoyclopropane-1-carboxylate deaminase depends on nutrient status of the plant. Can. J. Microbiol. 48:189-199.

Belimov A A, I C Dodd, V I Safronova, N Hontzeas, W J Davies (2007) Pseudomonas brassicacearum strain Am3 containing 1-aminocyclopropane-1-carboxylate deaminase can show both pathogenic and growth-promoting properties in its interaction with tomato. J. Exp. Bot. 58:1485-1496.

Blaha D, C Prigent-Combaret, M S Mirza, Y Moënne-Loccoz (2006) Phylogeny of the 1-aminocyclopropane-1-carboxylic acid deaminase-encoding gene acdS in phytobeneficial and pathogenic Proteobacteria and relation with strain biogeography. FEMS Microbiol. Ecol. 56:455-470.

Bleecker A B, H Kende (2000) Ethylene: a gaseous signal molecule in plants. Annu. Rev. Cell Develop. Biol. 16:1-18.

Burd G I, D G Dixon, B R Glick (1998) A plant growth-promoting bacterium that decreases nickel toxicity in seedlings. Appl. Environ. Microbiol. 64:3663-3668.

Burd G I, D G Dixon, B R Glick (2000) Plant growth-promoting bacteria that decrease heavy metal toxicity in plants. Can. J. Microbiol. 46: 237-245.

Castro-Sowinski S, Y Herschkovitz, Y Okon, E Jurkeyitch (2007) Effects of inoculation with plant growth-promoting rhizobacteria on resident rhizosphere microorganisms. FEMS Microbiol. Lett. 276:1-11.

Cheng Z, B P Duncker, B J McConkey, B R Glick (2008) Transcriptional regulation of ACC deaminase gene expression in Pseudomonas putida UW4. Can. J. Microbiol. 54:128-136.

Curl E A, B Truelove (1986) The Rhizosphere. Advanced Series in Agricultural Sciences, Vol. 15. Springer-Verlag, Berlin. 288 p.

Davison J (1988) Plant beneficial bacteria. Nature Biotech. 6:282-286.

Des Marais D L, T E Juenger (2010) Pleiotropy, plasticity, and the evolution of plant abiotic stress tolerance. Ann. N. Y. Acad. Sci. 1206:56-79.

Dobbelaere S, J Vanderleyden, Y Okon (2003) Plant Growth-Promoting effects of diazotrophs in the rhizosphere. Crit. Rev. Plant Sci. 22:107-149.

Esquivel-Cote R, R M Ramírez-Gama, G Tsuzuki-Reyes, A OrozcoSegovia, P Huante (2010) Azospirillum lipoferum containing 1-aminocyclopropane-1-carboxylic acid deaminase improves early growth of tomato seedlings under nitrogen deficiency. Plant Soil 337:65-75.

Gamalero E, G Berta, N Massa, B R Glick, G Lingua (2010) Interactions between Pseudomonas putida UW4 and Gigaspora rosea BEG9 and their consequences for the growth of cucumber under saltstress conditions. J. Appl. Microbiol. 108:236-245.

Glick B R (1995) The enhancement of plant growth by free-living bacteria. Can. J. Microbiol. 41:109-117.

Glick B R (2003) Phytoremediation: synergistic use of plants and bacteria to clean up the environment. Biotech. Adv. 21:383-393.

Glick B R (2004) Teamwork in phytoremediation. Genetically modified (GM) bacteria improve the degradation of toluene in plants. Nature Biotech. 22:526-527.

Glick B R (2005) Modulation of plant ethylene levels by the bacterial enzyme ACC deaminase. FEMS Microbiol. Lett. 251:1-7.

Glick B R, D M Karaturovic, P C Newell (1995) A novel procedure for rapid isolation of plant growth promoting pseudomonads. Can. J. Microbiol. 41:533-536.

Glick B R, D M Penrose, J Li (1998) A model for the lowering of plant ethylene concentrations by plant growth-promoting bacteria. J. Theor. Biol. 190:63-68.

Glick B R, B Todorovic, J Czarny, Z Cheng, J Duan, B McConkey (2007) Promotion of plant growth by bacterial ACC deaminase. Crit. Rev. Plant Sci. 26:227-242.

Grichko V P, B Filby, B R Glick (2000) Increased ability of transgenic plants expressing the bacterial enzyme ACC deaminase to accumulate $\mathrm{Cd}, \mathrm{Co}, \mathrm{Cu}, \mathrm{Ni}, \mathrm{Pb}$, and $\mathrm{Zn}$. J. Biotechnol. 81:45-53.

Grichko V P, B R Glick (2001a) Ethylene and flooding stress in plants. Plant Physiol. Biochem. 39:1-9.

Grichko V P, B R Glick (2001b) Amelioration of flooding stress by ACC deaminase-containing plant growth-promoting bacteria. Plant Physiol. Biochem. 39:11-17.

Holguín Z G, B R Glick (2001) Expression of the ACC deaminase gene from Enterobacter cloacae UW4 in Azospirillum brasilense. Microbial Ecol. 41:281-288.

Holguín Z G, B R Glick (2003) Transformation of Azospirillum brasilense Cd with an ACC deaminase gene from Enterobacter cloacae UW4 fused to the Tetr gene promoter improves its fitness and plant growth promoting ability. Microbial Ecol. 46:122-133. 
Hontzeas N, J Zoidakis, B R Glick, M M Abu-Omar (2004) Expression and characterization of 1-aminocyclopropane-1-carboxylate deaminase from rhizobacterium Pseudomonas putida UW4: a key enzyme in bacterial plant growth promotion. Bioch. Biophys. Acta 1703:11-19.

Hontzeas N, A O Richardson, A A Belimov, V I Safranova, M M AbuOmar, B R Glick (2005) Evidence for horizontal gene transfer (HGT) of ACC deaminase genes. Appl. Environ. Microbiol. 71:7556-7558.

Jing Y, Z He, X Yang (2007) Role of soil rhizobacteria in phytoremediation of heavy metal contaminated soils. J. Zhejiang Univ. Sci. B 8:192-207.

Kende H (1993) Ethylene biosynthesis. Annu. Rev. Plant Physiol. Plant Mol. Biol. 44:283-307.

Klee H J (1993) Ripening physiology of fruit from transgenic tomato (Lycopersicon esculentum) plants with reduced ethylene synthesis. Plant Physiol. 102:911-916.

Kloepper J W, S Tuzun, L Liu, G Wei (1993) Plant growth-promoting rhizobacteria as inducers of systemic disease resistance. In: Pest Management: Biological Based Technologies. R D Lumsdem, J L Vaughn (eds). Washington DC. American Chemical Society Books. pp:156-165.

Lynch J, K M Brown (1997) Ethylene and plant responses to nutritional stress. Physiol. Plant. 100:613-619.

Ma W, T C Charles, B R Glick (2004) Expression of an exogenous 1-aminocyclopropane-1-carboxylate deaminase gene in Sinorhizobium meliloti increases its ability to nodulate alfalfa. Appl. Environ. Microbiol.70:5891-5897.

McDonnell L, J M Plett, S Andersson-Gunneras, C Kozela, J Dugardeyn, D Van Der Straeten, B R Glick, B Sundberg, S Regan (2009) Ethylene levels are regulated by a plant encoded 1-aminocyclopropane-1-carboxylic acid deaminase. Physiol. Plant. 136:94-109.

Madhaiyan M, S Poonguzhali, J Ryu, T M Sa (2006) Regulation of ethylene levels in canola (Brassica campestris) by 1-aminocyclopropane-1-carboxylate deaminase-containing Methylobacterium fujisawaense. Planta 224:268-278.

Mayak S, T Tirosh, B R Glick (2004a) Plant growth-promoting bacteria that confer resistance to water stress in tomato and pepper. Plant Sci. 166:525-530

Mayak S, T Tirosh, B R Glick (2004b) Plant growth-promoting bacteria that confer resistance in tomato plants to salt stress. Plant Physiol. Biochem. 42:565-572.

Morgan P W, M C Drew (1997) Ethylene and plant responses to stress. Physiol. Plant. 100:620-630.

Nie L, S Shah, A Rashid, G I Burd, D G Dixon, B R Glick (2002) Phytoremediation of arsenate contaminated soil by transgenic canola and the plant growth-promoting bacterium Enterobacter cloacae CAL2. Plant Physiol. Biochem. 40:355-361.

Nukui N, K Minamisawa, S I Ayabe, T Aoki (2006) Expression of the 1-aminocyclopropane-1-carboxylic acid deaminase gene requires symbiotic nitrogen-fixing regulator gene nifA2 in Mesorhizobium loti MAFF303099. Appl. Environ. Microbiol. 72:4964-4969.

Penrose D M, B R Glick (2001) Levels of ACC and related compounds in exudates and extracts of canola seeds treated with ACC deaminase-containing plant growth-promoting bacteria. Can. J. Microbiol. 47:368-372.

Penrose D M, B R Glick (2003) Methods for isolating and characterizing ACC deaminase-containing plant growth-promoting rhizobacteria. Physiol. Plant. 118:10-15.
Plett J M, L McDonnell, S Regan (2009) Plant encoded 1-aminocyclopropane-1-carboxylic acid deaminase activity implicated in different aspects of plant development. Plant Signal. Behav. 4:1186-1189.

Prigent-Combaret C, D Blaha, J F Pothier, L Vial, M A Poirier, F Wisniewski-Dye, Y Moenne-Loccoz (2008) Physical organization and phylogenetic analysis of acdR as leucine-responsive regulator of the 1-aminocyclopropane-1-carboxylate deaminase gene acdS in phytobeneficial Azospirillum lipoferum 4B and other Proteobacteria. FEMS Microbiol. Ecol. 65:202-219.

Reed M L E, B G Warner, B R Glick (2005) Plant growth-promoting bacteria facilitate the growth of the common reed Phragmites australis in the presence of copper or polycyclic aromatic hydrocarbons. Curr. Microbiol. 51:425-429.

Rodríguez H, S Vessely, S Shah, B R Glick (2008) Effect of a niqueltolerant ACC deaminase-producing Pseudomonas strain on growth of nontransformed and transgenic canola plants. Curr. Microbiol. 57:170-174.

Robison M M, S Shah, B Tamot, K P Pauls, B A Moffatt, B R Glick (2001) Reduced symptoms of Verticillum wilt in transgenic tomato expressing a bacterial ACC deaminase. Mol. Plant Pathol. 2:135-145.

Saravanakumar D, R Samiyappan (2007) ACC deaminase from Pseudomonas fluorescens mediated saline resistance in groundnut (Arachis hypogea) plants. J. Appl. Microbiol. 102:1283-1292.

Shah J (2009) Plants under attack: systemic signals in defence. Cur. Op. Plant Biol. 12:459-464.

Shaharoona B, M Arshad, Z A Zahir, A Khalid (2006) Performance of Pseudomonas spp. containing ACC-deaminase for improving growth and yield of maize (Zea mays L.) in the presence of nitrogenous fertilizer. Soil Biol. Biochem. 38:2971-2975.

Shaharoona B, M Naveed, M Arshad, Z A Zahir (2008) Fertilizer-dependent efficiency of pseudomonads for improving growth, yield, and nutrient use efficiency of wheat (Triticum aestivum L.). Appl. Environ. Microbiol. 79:147-155.

Siddikee M A, P S Chauhan, R Anandham, G H Han, T Sa (2010) Isolation, characterization, and use for plant growth promotion under salt stress, of ACC deaminase-producing halotolerant bacteria derived from coast soil. J. Microbiol. Biotechnol. 20:1577-1584

Stearns J C, S Shah, B M Greenherg, D G Dixon, B R Glick (2005) Tolerance of transgenic canola expressing 1-aminocyclopropane1-carboxylic acid deaminase to growth inhibition by nickel. Plant Physiol. Biochem. 43:701-708.

Tahir M, M Arshad, M Naveed, Z A Zahir, B Shaharoona, R Ahmad (2006) Enrichment of recycled organic waste with $\mathrm{N}$ fertilizer and PGPR containing ACC-deaminase for improving growth and yield of tomato. Soil Environ. 25:105-112.

Ververidis P, P John (1991) Complete recovery in vitro of ethylene-forming enzyme. Phytochemistry 30:725-727.

Zahir Z A, M Arshad, W T Jr Frankenberger (2004) Plant growth promoting rhizobacteria: applications and perspectives in agriculture. Adv. Agron. 81:97-168.

Zhang Y F, L Y He, Z J Chen, Q Y Wang, M Qian, X F Sheng (2011) Characterization of ACC deaminase-producing endophytic bacteria isolated from copper-tolerant plants and their potential in promoting the growth and copper accumulation of Brassica napus. Chemosphere 83:57:62. 